\title{
Biology vs. Moral Objectivity
}

\author{
Armin Nikkhah Shirazi
}

\section{Introduction}

One way to characterize philosophy is by thinking of it as a field that concerns itself with the study of a collection of very hard to intractable problems that are left over after all the others which one could study have properly become subjects of other, distinct fields of study (Belot, 2009). To give but one example, for over 2000 years the study of what we now call 'physics' was properly considered a branch of philosophy, and indeed even called 'natural philosophy', until with the advent of Newton's framework it separated itself out as a distinct field.

A branch of philosophy which has so far resisted this sort of secession is ethics. In particular, philosophers concerned with moral problems have not normally used such methods as used in science to gain further insight into their questions of interest.

In 1986 the biologist Edward Wilson, in collaboration with the philosopher Michael Ruse, published an article in which they argued broadly that with the advent of a better understanding of the evolutionary origin and biological functioning of the human brain, one can apply principles derived from the field of biology in general, and evolutionary biology in particular, to the study of moral philosophy and hence transform it into an applied science (Ruse \& Wilson, 1986). Ruse and Wilson's article was one in a series of writings since at least 1975 in which Wilson argued that our understanding of ethics would improve in essential ways by considering it within the context of biology, or that ethics should be "biologicized” (Kitcher, 2006). One may consider this endeavor a part of sociobiology, where 'sociobiology' can be defined as "a research program that seeks to use evolutionary theory to account for significant social, psychological, and behavioral characteristics in various species" (Sober, 2000). The beginning of sociobiology is generally taken to be Wilson’s massive tome “Sociobiology: The new Synthesis” (Wilson, 1975). 
Among the most remarkable of the claims in the 1986 paper is that "Beliefs in extrasomatic moral truths...are wrong. Moral premises relate only to our physical nature and are the result of an idiosyncratic genetic history”.

If this claim is correct, then it means that we have no objective basis on which to judge any action as morally right or wrong; the best we can do is to frame our reasons for thinking or intuiting one way or another in terms of our 'idiosyncractic genetic history'. To the extent that this genetic history, and its influence on other relevant factors, such as our social and cultural background, shapes our attitudes regarding such actions, this position can be considered a form of emotivism. As a philosophical position, emotivism has consequences that one may consider highly unpalatable. Thus, to give an extreme example provided by Kitcher, "willfully torturing children must be seen as akin to...[having] bizarre gastronomic preferences” because the only reason we find such action morally repugnant, according to an emotivist, is that we find it emotionally repugnant, not because there is any intrinsic moral wrongness to this action as a matter of fact. Unsurprisingly, Wilson's arguments for his emotivist version of sociobiology proved to be highly controversial and provoked many hostile responses (Lewontin, 1984).

This paper will, after summarizing Ruse and Wilson's 1986 article, present an original defense of moral objectivism based on the idea that ethics and mathematics are more similar to one another than one might have supposed. It will then present two objections of which the second undercuts this defense and forces a direct assessment of one aspect of Ruse and Wilson's claim. It will be found that their claim is too sweeping to follow from the arguments they present, but that the possibility that it might be correct still exists.

\section{Ruse and Wilson's Moral Philosophy as Applied Science}

In their article, Ruse and Wilson begin by criticizing the fact that in much of moral philosophy, ethical premises are regarded as “directives independent of human evolution, with a claim to ideal, eternal truth.” They then lay out the premise of a naturalistic approach to ethics, according to which "Everything human, including the mind and culture, has a material base and originated during the evolution of the human genetic constitution and its interaction with the environment”. Next, they summarize how the process of natural selection drives evolution, pointing out that beyond healthy skepticism which should be afforded as it would to any other scientific theory, 
there is no doubt that natural selection is the correct scientific explanation of the mechanism for evolution. They note that human evolution, in particular, fully conforms to this explanation before presenting some specific biological examples in support of this view.

They then claim that our sense of 'right' and 'wrong' are "in fact brought about by ultimately biological processes" by means of epigenetic rules, which they define as "genetically based processes of development that predispose the individual to adopt one or a few forms of behaviors as opposed to others". The overall sequence they envision is that through mutation and selection ensembles of genes "prescribe epigenetic rules of mental development peculiar to the human species" under the influence of which "certain choices are made from among those conceivable” and which are finally "narrowed through contractual agreements and sanctification”.

A striking example for the influence of epigenetic rules they provide is the discontinuous perception of color change in humans under a continuous change of the wavelength of light. As another more relevant example within a moral context they present the prohibition on brothersister incest found in most cultures. They attribute to epigenetic selection rules a "sensitive period between birth and approximately six years" during which time exposure to other children “under conditions of close proximity” leads to an inability to form sexual bonds later. They argue that "lowered genetic fitness due to inbreeding led to the evolution of the juvenile sensitive period by means of natural selection; the inhibition experienced at sexual maturity led to prohibitions and cautionary myths against incest.”

Having presented these and several other examples as illustrations of their view that epigenetic rules underlie much if not all of the content of moral codes, they propose that "implicit in the scientific interpretation of moral behavior is...that there can be no genuine external ethical premises." They claim that therefore "It is thus entirely correct to say that ethical laws can be changed, at the deepest level, by genetic evolution.”

In the penultimate part of their essay, they attempt to address several objections that might be leveled against their view: They assert that biological altruism can still be compatible with genuine altruism, so long as one's epigenetic rules deceive one to think that it is morally right to be altruistic; that their thesis does not lead to moral relativism because "epigenetic rules are only relative to the species...not relative to the individual.”; and that the moral distinction between is 
and ought "in no way invalidates the evolutionary approach" because "moral codes are seen to be...legitimated by the illusion of objectivity.”

Finally, they articulate a vision in which "moral reasoning offers an exciting potential for empirical research and a new understanding of human behavior” and attempt to lay out a research program for the empirical study of morality from a biological point.

Ruse and Wilson's article is meant to lead to the conclusion that the intuition, probably shared by most humans, that an objective distinction between a moral right and wrong exists, is nothing but an illusion. The next section will present an argument in favor of the view that this distinction does exist independently from our or any other species.

\section{A Defense of Moral Objectivity}

It is probably fair to say that mathematical propositions are widely regarded as being correct or incorrect independent of the details and even the fact of our existence, and this view goes at least as far back as Plato. A moral objectivist espousing this view might strive to show that moral propositions are formally like mathematical propositions. If they are, then the view that certain

mathematical propositions can be regarded as correct or incorrect in an absolute sense would also seem to support the view that at least some moral propositions can be right or wrong in an absolute sense.

In this section, I wish to present an original argument to defend moral objectivism based on this strategy. This defense attempts to highlight the similarity between mathematical and moral systems by drawing three analogies between the two different systems.

The first analogy is based on the recognition that both systems permit the expression of distinctions that make it possible to impose some kind of ordering on the sets of objects under consideration. For simplicity, I will limit the discussion on the mathematical side to numbers, where the ordering relation is most precise and obvious. Then, just as it is coherent within a mathematical system to claim that one number can be either larger than, smaller than, or equal to another number, it is coherent within any reasonable moral system to claim at least for some set of actions that, say, one action can be either more morally right, less morally right, or equally morally right as compared to another action. It is true that in mathematics the distinction between numbers can generally be stated much more precisely than in the other system, but this 
difference seems rather secondary and may, at least in principle, be addressed, for example by a more precise definition of each action under consideration and what it entails.

One way one could minimize this difference to make mathematics more similar to a moral system is to imagine a mathematical system in which propositions, insofar as they refer to the distance from zero, instead applying to numbers, apply to number intervals. This introduces ambiguities into certain propositions which are usually absent in mathematics but are analogous to ambiguities that are quite common in ethics. For example, the question of whether the interval $[2,4]$ or the interval $[1,5]$ is farther from zero cannot be answered without specifying the method by which one arrives at the answer: If one considers the smallest number to be the determining factor in arriving at an answer, then [1,5] is closer, if one considers the largest number to be such, then $[2,4]$ is closer, and if one considers the average number, then both intervals are equally close. This seems to mirror quite closely an analogous requirement in many moral situations. For instance, the question of whether it is more evil to, say, steal an apple from a hungry rich man or an apple from a full poor man cannot be answered without specifying the method by which one arrives at an answer: If one considers satiety status to be the determining factor in arriving at a moral judgment, then it is more evil to steal from the hungry man than from the full man, if one considers financial status to be such, then it is more evil to steal from the poor man than from the rich man, and if one considers the value of the apple, then both acts are equally evil. It should also be pointed out that in neither the mathematical nor the ethical example the considerations mentioned exhaust all possible considerations one might bring to bear in arriving at an answer.

While the example just given is only a caricature of the kinds of moral problems one might seriously consider, it hopefully illustrates that moral and mathematical systems, at least to some extent, can be made similar as it pertains to distinctions that permit the imposition of an ordering. The second analogy depends on considering each branch of mathematics, such as the theory of numbers, as being comprised of several distinct subsystems, one for each set of numbers, rather than as a single unified system. Thus, one may consider the arithmetic of, say, quaternions, as distinct from that of complex numbers, and that of complex numbers as distinct from that of the real numbers, and so on. From this perspective, it is possible that certain propositions in one subsystem contradict propositions in another, provided one restricts one's consideration only to 
the context of a single subsystem. For instance, while within the context of the real numbers it is always incorrect to claim that the product of two numbers of equal sign is negative, it is quite possible that the product of two complex numbers with real parts of same sign has a negative real part. In fact, within the context of purely imaginary numbers, it is always correct to claim that the product of two numbers of same sign is negative (albeit the product is real). One might object that this is a simple consequence of the rules that define imaginary numbers, but consider that within the context of the set of real numbers, imaginary numbers do not exist. Hence, from within the context of real numbers, it remains incorrect to claim that the product of two numbers of equal sign can be negative.

How does this observation relate to moral systems? A prominent feature of moral philosophy is that there exist quite a number of distinct moral systems, and it is not uncommon that, say, a particular action which is morally right within the context of one moral system can be morally wrong within the context of another. For instance, consider a person who steals an apple from a full rich person to give it to a hungry poor person. A utilitarian might consider this to provide an overall higher utility compared to the situation in which the theft had not been committed, and thus judge the theft to be morally right. A moral absolutist, on the other hand, may consider the action to be morally wrong on the grounds that he considers theft to be wrong as a matter of principle.

In light of this simple example, interpreting mathematical subsystems as above draws out a similarity between moral and mathematical systems. In the example given it is true that the relationships between the different number systems are much more precise and well-defined than the relationships between the different kinds of moral systems, but this may at least in part reflect the greater complexity of the latter.

The third analogy focuses on the similarity of the effect of a contradiction on both kinds of systems. One way of regarding a mathematical system is to conceive of it axiomatically. Thus, one begins with a small number of unproven claims from which the entire framework is developed. An implicit aspect of an axiomatic approach is that it must include a prohibition on axioms that introduce a contradiction.

For instance, a number system in which $0=1$ is included as an axiom can no longer be considered as such. The reason is that the inclusion of such an axiom renders the system incoherent; the 
axiom could be used to show that any number is equal to any other number. For instance, one could show that $2=5$ by multiplying the 'equation' $0=1$ by 3 and then adding it twice it to $2=2$.

To apply this observation to moral systems, we must articulate a claim which plays a role that is analogous to the axiom ' $0=1$ '. This is not difficult, and the following statement, which I will call a moral axiom, will do: 'The action $\mathrm{X}$ is morally wrong and the action $\mathrm{X}$ is morally neutral'. Here, by 'morally neutral', I mean something that is neither morally right nor morally wrong. For example, one might consider the orbiting of the planets around the sun to be morally neutral.

To examine the effect of this contradiction on the moral system, let us substitute the concrete action 'stealing an apple' for X. Let us consider, then, a moral system which contains the moral axiom “Stealing an apple is morally wrong and stealing an apple is morally neutral”. Surely, if stealing one apple is morally wrong, then, everything else held equal, stealing two apples must be even more morally wrong, otherwise that would already call into question to what extent the moral system is capable of distinguishing between different moral actions. But if stealing one apple is morally neutral, then so must be stealing two apples. In short, an action which is morally more wrong than the original action to which the moral axiom app1ied also becomes morally neutral. How far can we take this? If stealing two apples is morally neutral, then so must be stealing three, four, and in fact all the apples in the world. Let us whimsically imagine that apples were the only kind of food edible to humans.

Stealing all the apples would then cause everyone to starve to death. One can imagine few evils greater than causing every living person to starve to death, yet in this moral system, this action is morally neutral. At the same time, this action is also morally wrong within this moral system, at least to the extent one can establish a relation between the morality of stealing an apple and that of causing someone (or everyone) to starve to death. One admittedly crude way of doing this would be to put a monetary value on apples and human lives in order to compare the moral harm of stealing an apple directly to that of causing someone to starve to death. In exact analogy to the fact that ' $0=1$ ' can be used to show that any number is equal to any other number, the moral axiom "Stealing an apple is morally wrong and stealing an apple is morally neutral" can then be used show that any morally wrong action is as morally neutral as any other morally wrong action, again, provided the actions can be shown to bear a relation to the action to which the selfcontradictory moral axiom refers. To summarize, the introduction of a contradiction has caused 
the moral system to become incoherent, just as the introduction of a contradiction caused the number system to become incoherent.

Why is it important to point this out? Because it may well be a general impression (and certainly one that Ruse and Wilson share) that moral systems are, from an intrinsic point of view, entirely arbitrary. To the extent that mathematics is not considered to be arbitrary, this would support the view that ethics does not have the same claim to an existence independent of our species. The third analogy is meant to illustrate that while moral systems may certainly be more flexible than mathematical systems-one might, for instance, conceive of a moral system in which stealing an apple is morally more wrong than killing a person- the flexibility is not without limits, and hence moral systems are not entirely arbitrary. In fact, the above discussion demonstrates that the constraint on the arbitrariness of a moral system is exactly analogous to a constraint on the arbitrariness of an arithmetic system. Just as the introduction of ' $0=1$ ' causes a number system to collapse, the introduction of a seemingly innocuous moral axiom like "Stealing an apple is morally wrong and stealing an apple is morally neutral" can cause a moral system to collapse.

\section{An Objection Based on Dissimilarities between Mathematics and Ethics}

A first line of attack against the defense given is to acknowledge the analogies between mathematical and moral systems, and then attempt to undermine them by pointing out how they are in other important respects quite disanalogous. A stark reflection of the fact that there are, in fact, dissimilarities between them is that disagreements over mathematical matters between mathematicians tend to evaporate once the basic assumptions and terms, as well as the exact steps involved in the arguments are clarified, whereas such a dissolution of disagreement remains all too elusive in discussions on moral matters.

To address this objection, we need to analyze the differences between mathematics and morality and examine to what extent these really do undercut the defense given above. I see at least three: The first concerns the fact that mathematical matters are much further removed from immediate human affairs than moral matters are, and this greatly facilitates dispassionate discourse in the former. Discussions on moral subjects, especially when they involve situations associated with extreme suffering or injustice, can quite easily become emotionally laden. Indeed, one may even go so far as to argue that it is not regarded as inappropriate not to maintain a totally emotionally 
unaffected attitude in such discussions on pains of giving the impression of being a psychopath, or at least an immoral person. In contrast, a mathematician who displays emotional attachments to certain mathematical positions would very likely be labeled as eccentric, if not outright biased. While emotional commitments to certain moral positions undoubtedly contribute to difficulties in attaining agreement in discussions of moral matters, this does not reflect a fault of the defense of moral objectivity but of the way in which moral discussions may be conducted.

There are simple and sensible ground rules which go a long way toward facilitating productive discussion on any topic, but which should be heeded especially conscientiously when it comes to discussing moral matters, such as: don't judge your opponents based on the positions they take; don't make challenges to your positions personal; try to understand why your opponents hold the views they do; value being free from misconceptions more than being right.

The second dissimilarity concerns the extent to which each system represents something beyond itself. Pure mathematics is, rather uniquely, characterized by the fact that it represents nothing beyond itself: in mathematics, the map is the territory. As a result, mathematical systems, as representations, are perfect. Moral systems, on the other hand, are models, simplified and imperfect representations of human interactions on the moral plane which can and often do fail to take into account salient nuances of the situations they are meant to represent, a problem which gets only worse with increased complexity of the situation under consideration. Furthermore, different people may be prejudiced to attach moral values differently to both those aspects which are accounted for in the model and those left unaccounted, thereby increasing the danger of introducing hidden factors into discourse which influence it but are never brought out into the open. Finally, moral arguments expressed in a natural language like English tend to be imprecise, eloquence may conceal sophistry and vagueness may obscure faulty reasoning.

All of these factors tend to undercut the ability to reach an agreement in a discussion of moral matters, but they do not undercut the defense given, for, once more, they represent not shortcomings of the mentioned analogies between mathematics and moral systems but of the way in which moral discourse may be conducted.

There are tools to help address at least some of these problems: For example, the formalism of modal logic has been adopted to create a formal system called deontic logic, which permits one 
to express within a formal language notions of obligatory and permissible actions. Arguments expressed within this formalism seem especially similar to mathematical arguments. Unfortunately, deontic logic is known to give rise to certain kinds of “paradoxes”, e.g. logically valid arguments which express counterintuitive, morally unsavory, or even contradictory conclusions derived from reasonable premises (Meyer et. al., 1994). Nevertheless, it points to an ideal according to which moral matters could be discussed quite similarly to mathematical ones, and at least some of the shortcomings of deontic logic can be attributed to its lack of sophistication in capturing shades of meaning in natural language. Use of additional formal tools available to other systems of logic, such as non-monotonic logic, and innovations in the field of linguistics, in particular, could help to develop more comprehensive formal languages suitable for the development and productive discussion of moral systems.

The third dissimilarity I see is that mathematical propositions, to the extent that they do not refer to our actual world, are timeless; a theorem proved remains a theorem for all eternity. Moral systems, to the extent they are meant to be useful as guides of moral conduct, cannot be so, at least not in their entirety, because prevailing moral standards may and in the past often did change. Furthermore, moral considerations in some situations may become obsolete while in others they may not even be meaningful yet. For instance, the moral aspects of medieval feudal systems are, as a practical matter, no longer relevant in the $21^{\text {st }}$ century, whereas at the time that they were relevant, moral aspects of cybercrimes would have been devoid of meaning.

However, this distinction between mathematics and moral systems is not very unlike the distinction between mathematics and science, and there the changing nature of the applicability of individual scientific systems to worldly matters does not undercut the possibility that one can still talk about an objective difference between correct and incorrect propositions on a nonarbitrary basis within the context of those systems. Indeed, one can sharpen the similarity between scientific and moral systems in this regard by limiting one's consideration to their respective most fundamental laws or principles: Just as the obsoleteness of the applicability of Newton's second law to, say, mammoths does nothing to invalidate the law itself within classical mechanics, the obsoleteness of the applicability of the immorality of curtailing someone's freedom against their will-supposing that is taken as a fundamental moral principle in a given system-to feudal serfdom does nothing to invalidate that principle within the moral system. 
It is the case, however, that periodically worldviews in science undergo sometimes significant changes, and it has been argued that such changes should be elevated to the level of revolutions in which a prevailing scientific paradigm is replaced by a new one (Kuhn, 1962). For example, we now know that classical mechanics is, strictly speaking, false and merely an approximation to relativistic mechanics.

This raises the question of whether there could not be similar "moral revolutions" which undermine the purported analogy between ethics and mathematics. For example, we know that $1+1=2$ will always be true, can we be similarly certain that a fundamental moral principle like, say, the golden rule will always hold? And if we cannot be certain of that, how can we still uphold the notion that ethics is analogous to mathematics?

To address this, let me briefly consider the notion of scientific revolutions in greater detail: Though Newtonian mechanics is strictly false, it is still usually the subject of the first physics course taught in at any university. That is because there exists a rather sizable domain of applicability within which it can be considered to be, for all practical purposes, correct. Here, "for all practical purposes" means that the observational consequences of the difference between the predictions of Newtonian and relativistic mechanics are in most cases negligible. Thus, for physicists the net effect of this revolution has been to shrink the domain of applicability of the former from universal to a smaller non-universal one circumscribed by value ranges for a specific parameter (low relative velocities, in this context).

It is not difficult to construct something analogous for ethics. For example, let us consider the golden rule which, it is probably fair to say, is widely considered as universally applicable a moral principle as any. In a commonly quoted version it says:

\section{Do unto others as you want to be done by}

The basic assumption behind it is that others have the same preferences for how they wish to be treated as we do. But now consider how extremely improbable it is that two people will have perfectly identical predilections for treatment by others, given the myriad of possible situations that human experience can offer. To explicate by example, it is probably true for most people that one way in which they want to be treated is that others be honest to them. But for each person there surely is a set of possible circumstances which, were they to occur, would negate 
that preference: Say, if being honest causes discomfort to oneself or the other person to varying degrees, and further depending on things like the subject matter, the setting of the communication, one's mood and which person is involved.

It should at least in principle be possible to construct an infinite set of such circumstances (a possible way to do this would be to define something like degree of discomfort as a continuous parameter), and since there have only been a finite number of people in the world, it is overwhelmingly unlikely that there are even just two people in all of human history who share identical predilections for being treated honestly under all possible circumstances. But that implies that it is overwhelmingly likely that the basic assumption on which the golden rule is based is, strictly speaking, false!

It turns out that there is a version that avoids this problem, and it is due to Popper (Popper, 1945):

Do unto others, wherever reasonable, as they want to be done by

Popper's variant does not depend on the assumption that others have the same preferences as we do (though it is based on other assumptions that could be, strictly speaking, false, like the assumption that we can accurately know how someone else wants to be treated). Nevertheless, one can consider a moral system based on Popper's variant as the moral analog of the relativistic correction to Newtonian mechanics in the sense that if we do permit the assumption that at least in some cases two people can have substantially similar preferences for how they want to be treated, then in those cases it essentially reduces to the golden rule.

In spite of what we just found, the golden rule can be justified as a good guide for moral conduct because for all practical purposes the assumption on which it is based is correct. Here, "for all practical purposes” means that the consequences of the difference in preference between people in how they like to be treated under all possible circumstances is in most cases negligible. The net effect of Popper's variant is to shrink the domain of the golden rule from universal applicability to one that only includes situations in which one's preference for being treated a certain way is not substantially different from that of others.

The bottom line is this: Yes, it must be conceded that the defense of moral objectivism given fails to support the notion that ethics is like mathematics with respect to the eternal validity of 
basic principles. But the way in which ethics falls short here is analogous to the way in which science falls short compared to mathematics, yet in science this shortcoming does not negate the ability to distinguish between correct and incorrect propositions on a non-arbitrary basis. Hence, if the shortcomings are really analogous, then they should not negate the ability to distinguish between correct and incorrect propositions on a non-arbitrary basis in ethics, either. But that implies an existence of ethics independent of our existence.

In short, the objection based on dissimilarities between mathematics and ethics based on the three arguments given here seems largely unsuccessful. However, it is possible to mount a much more potent challenge.

\section{An Objection Based on Denying the Independent Existence of Mathematics}

The argument in defense of moral objectivity attempts to show that ethics is more similar to mathematics than may be commonly supposed. Therefore, if one considers the existence of mathematics to be independent of our existence, then this similarity suggests that the existence of ethics is also independent of our existence.

An advocate of Ruse and Wilson's views could object to this argument by denying that mathematics has an existence independent of us; that even mathematics, being, as it is, a part of everything human and therefore having a material base, is a byproduct of our epigenetic rules. If that is true, then it does not matter how similar ethics is to mathematics; in that case, even proof of an isomorphism between the two is utterly useless in demonstrating that there is an objective distinction between right and wrong. Perhaps not too many would argue that mathematics owes its existence solely to the existence of our species, but those who do have a powerful objection that renders the defense given in support of moral objectivism irrelevant. Moreover, this is not just a hypothetical position to take: there is at least one school of thought in mathematics, namely intuitionism, which is explicitly based on the assumption that mathematics has no independent existence of its own but is purely the product of constructive human mental activities.

This forces one then to directly examine the merits of the arguments presented in Ruse and Wilson's paper. Even barring a defense of moral objectivity as presented above, I do not think it follows that their arguments establish that there is no ethical distinction between right and wrong that is external to us. More specifically, I believe that they make an unwarranted leap when they 
conclude from the fact that many animal species perform acts, such as cannibalism, incest, mutual eating of feces and so on, which are morally repugnant from a human perspective, that alien intelligent species might conceivably find those same actions to be highly moral. The leap seems unwarranted to me because it ignores the following important consideration:

If any of the animals which Wilson uses as his model for the morally repugnant actions were capable of having sufficiently sophisticated moral systems and performed these from an anthropological perspective morally repugnant actions because they found them to be highly moral, then his assertion could in fact be correct. Alas, humans are so far the only species known to have developed elaborate moral systems; the motivations of most other animals, for all we know, have absolutely nothing to do with morality; if some insects commit parricide, it seems safe to assume, they do not do so because they consider it the moral thing to do, and so his comparison of these animals with any species that has the capacity for making moral distinctions strikes one as analogous to comparing apples with oranges.

This has a dire consequence for trying to extrapolate his findings to scenarios that involve intelligent alien species who, for moral reasons that were epigenetically determined, perform actions that seem morally repugnant to us. The evidence given by Ruse and Wilson only supports that it is possibly the case that it is possible that, say, moral baby-eating aliens could exist. However, their conclusion is stronger than this. What their claim amounts to is that it is necessarily the case that it is possible that moral baby eating aliens could exist.

That is what is implied by the assertion that there is no extrasomatic basis for ethics, for in that case the possibility of evolution leading by means of epigenetic rules to intelligent species that can formulate any kind of moral system whatsoever, including one that sanctions babyeating, must necessarily be left open, as it is not constrained in any other way.

Although the distinction is subtle, it still renders their claim more sweeping than what is warranted by the arguments they give, and hence their conclusion does not follow. It is, however, interesting to consider what it would take to correct their argument, and a brief consideration of that will conclude this article. 


\section{The Central Challenge of Biologicizing Ethics}

That Ruse and Wilson's conclusion does not follow from their premises does not necessarily prove that their main overarching thesis, that ethics can be studied empirically using the tools of biology, is mistaken. In fact, I agree with this thesis in that I believe that it is at least in principle possible to do so.

The central obstacle that I see to doing that is this: empirically, the study of evolutionary biology in its present state is by itself insufficient to show that there is no extrasomatic distinction between a moral right and wrong because what is needed to show this is actual contact with and observation of at least one other species that

- follows totally different epigenetic rules than the ones humans follow, and

- $\quad$ which has developed a capacity for making moral distinctions that are at a level comparable to that of humans

Evolutionary biology thus far can only provide us with examples of species which either satisfy the first criterion (e.g. insects) or the second (i.e. humans), but not both.

It seems highly likely that if a species existed on earth which satisfied both criteria, it would have been discovered by now and would have lent itself as an object of investigation to study the question of whether biology is in fact the sole determinant of morality or not. Given the apparent absence of such a species on our planet, our next best hope might be contact with an extraterrestrial intelligence, which itself seems highly unlikely. An interesting consequence of such an event, if it were to ever happen and lead to the sort of investigations Ruse and Wilson envision, may well be that ethics does in fact split from philosophy to become an empirical field, leaving philosophy with even fewer tractable problems. 
Belot, G. (2009). Philosophy of Physics: Why Bother? Departmental Colloquium. Ann Arbor: University of Michigan, Department of Physics.

Kitcher, P.(2006) Four Ways of "Biologicizing" Ethics. In E. Sober, Conceptual Issues in Evolutionary Biology (pp. 575-586). Cambridge: Bradford Books.

Kuhn, T. (1962) The Structure of Scientific Revolutions. Chicago:University of Chicago Press Lewontin, R.(1984). Not in our Genes. New York: Pantheon Books.

Meyer J., Dignum F. \& R. Wieringa (1994) The Paradoxes of Deontic Logic Revisited: A Computer Science perspective Utrecht: Utrecht university

Popper, K. (1945) The Open Society and its Enemies, Vol. 2.London: Routledge

Ruse, M. \& Wilson, E. (1986). Moral Philosophy as Applied Science. Philosophy, 173-192

Sober, E. (2000) Philosophy of Biology. Boulder: Westview Press.

Wilson, E (1975) Sociobiology: The new Synthesis. Cambridge: Harvard University Press. 